NASA/CR-955- $207146 \quad$ NAG8-289

\title{
DISK-CORONA MODEL OF ACTIVE GALAC
NUCLEI WITH NONTHERMAL PAIRS
}

TIC $/ N-92-C K$

CWAIVED

067310

\section{SACHIKO TSURUTA and MICHAEL KELLEN}





\title{
DISK-CORONA MODEL OF ACTIVE GALACTIC NUCLEI WITH NONTHERMAL PAIRS
}

\author{
Sachiko Tsuruta and Michael. Ktiti.ien \\ Muntana State University, Department of Physics, Bozeman, MT 59717-0350 \\ Received 1995 February 21 ; accepted 1995 August 17
}

\begin{abstract}
As a promising model for the X-ray emission from radio-quiet quasars and Seyfert 1 nuclei, we present a nonthermal disk-corona model, where soft photons from a disk are Comptonized by the nonthermal electronpositron pairs in a coronal region above the disk. Various characteristics of our model are qualitatively similar to the homogeneous, spherical, nonthermal pair models previously studied, but the important difference is that in our disk-corona model $\gamma$-ray depletion is far more efficient, and, moreover, the $\gamma$-ray annihilation line is much less prominent. Consequently, this model naturally satisfies the observed constraints on active galactic nuclei.

Subject headings: accretion, accretion disks - galaxies: Seyfert - gamma rays: theory - radiation mechanisms: nonthermal — radiative transfer - X-rays: general
\end{abstract}

\section{INTRODUCTION}

Among various models suggested for the roughly power-law $X$-ray spectrum from a class of active galactic nuclei (AGNs) represented by type 1 Seyfert nuclei and radio-quiet quasars, the most promising appears to be the Comptonization of soft photons by energetic electron-positron $\left(e^{-}-e^{+}\right)$pairs. Pairs could be produced thermally (e.g., Sunyaev \& Titarchuk 1985; Lightman 1982) or nonthermally (c.g., Guilbert, Fabian, \& Rees 1983, hereafter GFR83; Fabian et al. 1986; Lightman \& Zdziarski 1987, hereafter LZ87; Done \& Fabian 1989, hereafter DF89; Zdziarski et al. 1990, hereafter Z90; Coppi \& Blandford 1990, hereafter CB90; Zdziarski \& Coppi 1991, hereafter ZC91). In essentially all of the nonthermal models investigated to date, a homogencous spherical geometry is adopted, where both soft and hard components are uniformly distributed within a spherical region. These types of models can naturally explain many aspects of the observed spectrum, but pose a serious problem in the sense that they produce a strong $\gamma$-ray annihilation line which has not been observed (see figures in, e.g., LZ87, DF89, and Z90). In our very recent work (Kellen, Tsuruta \& Tritz 1995, hereafter KTT95) a possible geometrical effect is introduced to the nonthermal pair-cascade model. In this communication we apply an improved version of the testing methods developed by DF89 to our nonthermal disk-corona model and demonstrate that our model more naturally satisfies the observed $\gamma$-ray constraints.

\section{PHYSICAL MODEL AND METHOD OF SOLUTION}

A Compton model involving $e^{-}-e^{+}$pairs generally consists of two components, the soft and hard photons (see, e.g., GFR83 and DF89). In our model a planar-surface source, such as a disk, emits the soft photons. It also emits $\gamma$-rays which initiate a pair cascade in the space above the source, and a pair corona results. Detailed calculations of the structure of the resulting pair-photon corona and of the spectral reprocessing are carried out in KTT95. Our basic equations and approach are similar to those in LZ87 and DF89, but the input microphysics has been considerably updated and improved, mostly by utilizing the work of $\mathrm{CB} 90$ and $\mathrm{ZC} 91$. Also, homogeneous spherical radiative transfer equations are replaced by a system describing radial out-streaming of radiation from the source. The major difference between the earlier homogeneous spher- ical model (hereafter referred to as "HS Model") and ours is that, in our model, the primary seed photon injection (both soft and hard) is confined to the region near the disk surface, while in HS Model it is distributed uniformly throughout the entire spherical emitting region. We consider that our model is physically more realistic, because in reality the efficient MHD acceleration of electrons to highly relativistic regimes, which in turn will produce the hard $l_{h}$ component as, e.g., synchrotron photons, probably takes place very close to the accretion disk surface (e.g., Galeev, Rosner, \& Vaiana 1979).

For the primary spectrum of soft photons emitted from the disk, our code will accept arbitrary spectra within the range of dimensionless photon energies $10^{-8}<x<2 \times 10^{4}$, where $x=h v / m_{i} c^{2}$. As a simple model for the so-called UV bump, which exists in most radio-quict quasars and Seyfert 1 nuclei, we assume a primary soft photon source spectrum given by the specific flux distribution $F_{\mathrm{c}}(x)$ (ergs $\mathrm{cm}^{-2} \mathrm{~s}^{-1}$ per unit $x$ ), which is proportional to a Planck distribution at temperature $T_{d i}$. A characteristic soft energy $x_{s}=2.8 k T_{d} / m_{c} c^{2}$ is defined at the peak of the soft emission. This simplification may affect the realistic modeling of the soft X-ray excess region. However, our main concern in this paper is the high- $\gamma$-ray region, and, hence, this simplification will not affect our major conclusions. The compactness parameter corresponding to soft emission is defined by $l_{s}=L_{s} \sigma_{\mathrm{T}} / R m_{r} c^{3}$, where $L_{s}=4 \pi R^{2} \int F_{\mathrm{s}}(x) d x$ is the soft luminosity and $\sigma_{r}$ is the Thomson cross section. ( $R$ is the effective radius of the emission region.) Substantial pair production requires that a sufficiently strong flux of hard $(x \geqslant 1)$ photons be present. Various authors have argued that accretion flows are natural sites for $\gamma$-ray production, through the presence of one or more particle acceleration mechanisms, such as shocks, magnetic reconnection, etc. It has been found that these relativistic particle accelerations generally produce power-law radiation (e.g., GFR83, LZ87, and DF89). Therefore, a "hard" primary spectral component, $F_{h}(x)$, is generally modeled as a power law, with the spectral energy index $\alpha_{0}$ and extending from the soft energy $x_{s}$ to a maximum emitted energy $x_{m}$. The compactness associated with hard emission is defined as $l_{h}=L_{h} \sigma_{\mathrm{T}} / R m_{c} \mathrm{c}^{3}$, where $L_{h}=4 \pi R^{2} \int F_{h}(x) d x$ is the hard luminosity. The relative strength of the soft and hard emission is set by the model parameter $l_{s} / l_{h 2}$. The total compactness of the source is $l=L \sigma_{\mathrm{T}} / R m_{c} c^{3}$, where $l=l_{s}+l_{h}$ and $L=L_{s}+L_{h}$ is the total luminosity. 


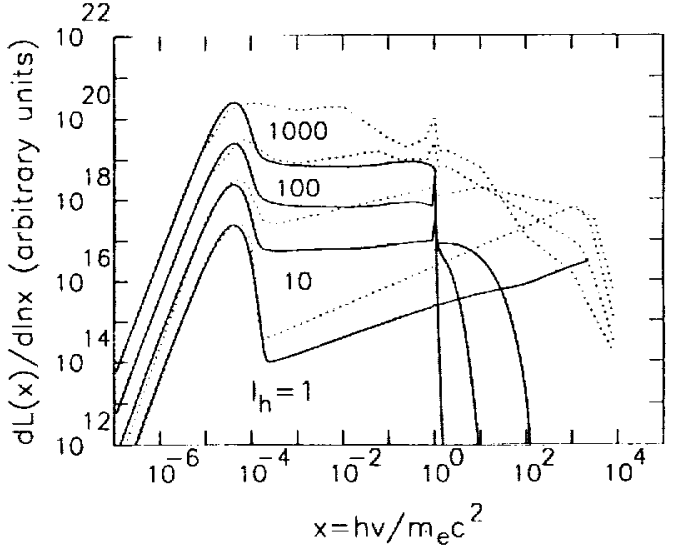

Fici. 1.-Emergent spectra are shown for power-law photon injection (solid cunces), with $x_{3}=3 \times 10^{5}, x_{m}=2.25 \times 10^{3}, \alpha_{0}=0.5, l_{j} / l_{h}=0.25$, and $l_{h}=\{1$, $10,100,1000\}$. The dotted curves reproduce the results of Fig. 2 in LZ87, roughly for the samc parameter ranges.

We treat radiative transfer by modeling the surface cascade as a shower atmosphere in which radiation streams outward through the pair plasma (see Tritz 1990, hereafter T90). This and some other simplifying assumptions were relaxed in T90, where the general case of arbitrarily anisotropic atmospheres is investigated. However, we have found that the present results agree qualitatively with the more exact results of T90, within a factor of $\sim 2$. The boundary conditions at the emission surface arc obtained from the primary emission fluxes. For both the boundary conditions and the various rate cquations which enter the photon continuity equation, the improved methods and equations in CB90 are adopted. For each photon energy $x$, the photon continuity equation is integrated from $h=0$ to typically $h=R_{i}$, where $R_{i}$ is the effective disk radius. At each $h$ the electron continuity equation is then integrated to obtain the nonthermal-pair density. The thermal-pair density and temperature are obtained by requiring that pair creation and annihilation be in equilibrium and the thermal population be in equilibrium with the radiation field. Hence, photon densities at each height $h$ are sufficient information to calculate the pair distribution at $h$, which consists of the nonthermal-pair density $N(\gamma, h)$ (electrons and positrons per unit volume per unit Lorentz factor $\gamma$ ) and the density $N_{0}(h)$ and temperature $T_{0}(h)$ of thermalized pairs. A more exact iteration method was adopted in T90, but we obtained similar results. The reason is that, owing to the absence of primary seed photon injection except at the base of the coronal region, no energy dissipation takes place in the whole coronal space except at the base, and also that the optical depth is much less than in the HS case (see § 4). Consequently, the contribution from the matter and radiation above $h$ is not significant. In view of the excessive computing requirements needed for the exact approach and the practical usefulness of the current simplified method, we conclude that the present approach is justified. Further details on the equations and methods are found in T90, KTT95, LZ87, DF89, and CB90.

\section{RESULTS AND COMPARISON WITII OBSERVATION}

The solid curves in Figure 1 show emergent spectra from a representative model with parameters chosen for comparison with figures in LZ87 and DF89. Injection parameters are chosen as $x_{s}=3 \times 10^{5}, l_{s} l_{h}=0.25, x_{m}=2.25 \times 10^{3}, \alpha_{0}=0.5$, and $l_{h}=\{1,10,100,1000\}$. Various spectral behavior noted in HS Model (e.g., LZ87, DF89, Z90, and ZC91) is seen in our disk-corona results as well. On the other hand, a comparison of the $\gamma$-ray region in our results with that of HS Model illustrates important differences. In Figure 1 the dotted curves show the emergent spectra from the roughly equivalent models in Figure 2 of LZ87. See KTT95 and T90 for detailed comparison of photon and electron injection parameters. The main quantitative difference (e.g., the slope and intensity of the power-law part of the spectra at a given $l_{h}$ ) is due to the improved microphysics we adopted. The major important differences to be noted are (i) the $\gamma$-ray annihilation line is much weaker for higher $l_{h}$, and (ii) depletion of the higher energy $\gamma$-rays is significantly more enhanced in our diskcorona model than in HS Model.

In order to conduct the observational tests on our model, the general approach of DF89 and LZ87 is adopted, with the following important revisions. When DF89 and LZ87 conducted their observational tests, one constraint was that the $\mathrm{X}$-ray energy index $\alpha_{x}$ be $0.1-1$. The recent development within the last few years, in both observation and theory, however, indicates that the observed X-ray energy index for $\gtrsim 2 \mathrm{keV}$ is close to 0.7 , and that this $\alpha_{x}$ is obtained as the final value for the spectrum reprocessed further through reflection by the cold matter, if the index of the intrinsic spectrum emerging from the pair cascade is $\sim 0.7-1$. Hence, the following updated constraints are applied to our model in order to delineate a parameter space in which our model is consistent with observations, Our first constraint, therefore, is that the $2-10 \mathrm{keV}$ index of our emergent spectra (from the pair atmosphere before reflection) be in the range $0.7<\alpha_{x}<1$. In our emergent spectra, we calculate $\alpha_{x}$ from the slope of a power-law fit to this energy band. Second, the ratio of UV luminosity to $\mathrm{X}$-ray luminosity, $l_{\mathrm{s}} / l_{\mathrm{x}}$, is restricted to the conservative range of $1<l_{s} / l_{x}<300$. Our upper limit is somewhat higher than the value adopted by DF89, because we take into account the uncertainty in the size of the UV bump. (Here the $\mathrm{X}$-ray compactness parameter is defined as $l_{\mathrm{x}}=L_{\mathrm{x}} \sigma_{\mathrm{v}} / R m_{\mathrm{c}} c^{3}$, where $L_{\mathrm{x}}$ is the $\mathrm{X}$-ray luminosity in the $2-10 \mathrm{keV}$ range.) The soft compactness parameter $l_{s}$ is a model parameter, while $l_{\mathrm{x}}$ is obtained by integrating energy flux over $2-10 \mathrm{keV}$. Third, observations of the $\gamma$-ray background provide an upper limit to the strength of the $1-100 \mathrm{MeV}$ band for a given $2-10 \mathrm{keV}$ flux. The $\gamma$-ray excess is defined as the ratio of the $1-100 \mathrm{MeV}$ encrgy flux to the maximal value allowed by $\boldsymbol{\alpha}_{\boldsymbol{x}}$. To be consistent with the $\gamma$-ray background measurements, AGNs in general must exhibit excesses less than or equal to unity. The fourth constraint is a maximum strength of the annihilation line required by the failure to detect it in observed spectra. Line strength is measured in terms of its equivalent width, $\mathrm{EW}$ (keV). Following DF89, if it is assumed that turbulent velocities in the system reach $0.3 c$, then annihilation lines must have EW $<300 \mathrm{keV}$ to remain undetected.

The results of our testing are summarized in Figures 2-4. The dotted curves represent a relatively high-energy injection capable of producing several generations of pairs, with $x_{s}=10^{4}$ and $x_{m}=1.333 \times 10^{4}$, while the solid curves represent a low-energy injection capable of producing one gencration of pairs, with $x_{s}=10^{\mathrm{s}}$ and $x_{m}=13.33$. In both cases $\alpha_{0}=0.5$ and $0.1<l_{h}<7 \times 10^{3}$. These models correspond roughly to the dotted and solid curves, respectively, in DF89. Figure 2 shows the $\gamma$-ray excess plotted against the EW of the annihilation line, for $0.1<l_{s} / l_{h}<10$. The acceptable region lies below the $\gamma$-ray excess $=1$ line and to the left of the $\mathbf{E W}=300 \mathrm{keV}$ line. The part of the curves which lies outside this acceptable rcgion corresponds to $l_{h}<10$. Hence we conclude that all of the parameter values for $l_{h}>10$ yield models which are consistent with these two constraints. Figure 


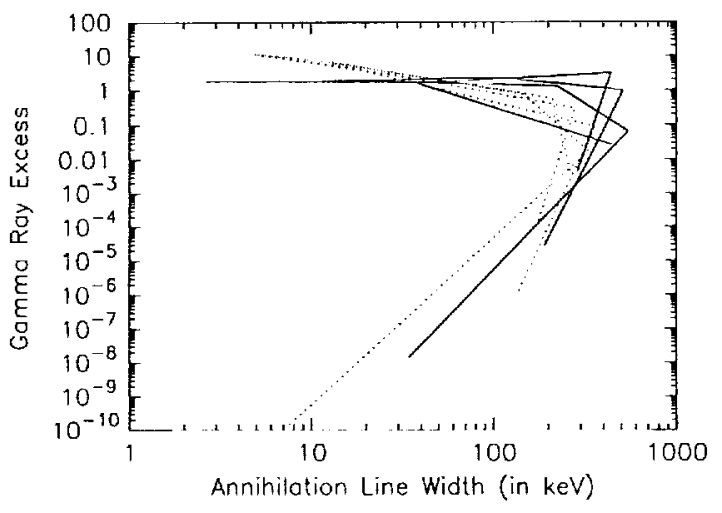

FIci. 2.-The $y$-ray excess is plotted against the $\mathbf{E W}$ of the annihilation line, for $0.1<l_{s} / l_{h}<10,0.1<l_{h}<7 \times 10^{3}$, and $\alpha_{0}=0.5$. The solid-line models indicate $x_{s}=10^{-5}$ and $x_{m}=13.33$, while the dotted-line models indicate $x_{s}=10^{-4}$ and $x_{m}=1.333 \times 10^{4}$.

3 shows the $\gamma$-ray excess plotted against $l_{j} / l_{x}$. The numbers attached to the curves refer to the values of $I_{s} / I_{h}$. To be consistent with the associated constraints, models must lie in the region below the excess = unity line, and between the $l_{s} / l_{\mathrm{x}}=1$ and $l_{s} / l_{\mathrm{x}}=300$ lines. We note that, again, the $\gamma$-ray excess constraint is satisfied by all of the parameter combinations when $l_{h}>10$. Figure 4 shows the $\mathrm{X}$-ray spectral energy index $\alpha_{x}$ plotted against $l_{s} / l_{x}$ (with the same notation as in Figure 3), and indicates that the observational constraint $0.7<\boldsymbol{\alpha}_{x}<1$ is satisfied for a significant range of our runs. From Figures 2-4 together, we find that successful models, consistent with all constraints, are produced by the following wide range of injection parameters: $0.1 \leqslant l_{s} / h_{h} \lesssim 5$ and $10 \leqq l_{h}$.

\section{DISCUSSION AND CONCLUDING REMARKS}

DF89 found that no homogeneous spherical (HS) injection model robustly satisfies all constraints. They did not take into account the effect of reflection. By including the reflection by cold matter, Z90 claim that this problem is solved, because the correct emergent spectral index should be larger. Then, since larger $\alpha_{x}$ corresponds to larger $l_{h}$ with more enhanced highenergy $\gamma$-ray depletion, the $\gamma$-ray excess no longer appears. However, with closer examinations, we note that, paradoxically, in their HS Model the situation which solves the $\gamma$-ray excess problem makes the annihilation-line problem even worse. This is because the larger $l_{h}$, which solves the $\gamma$-ray

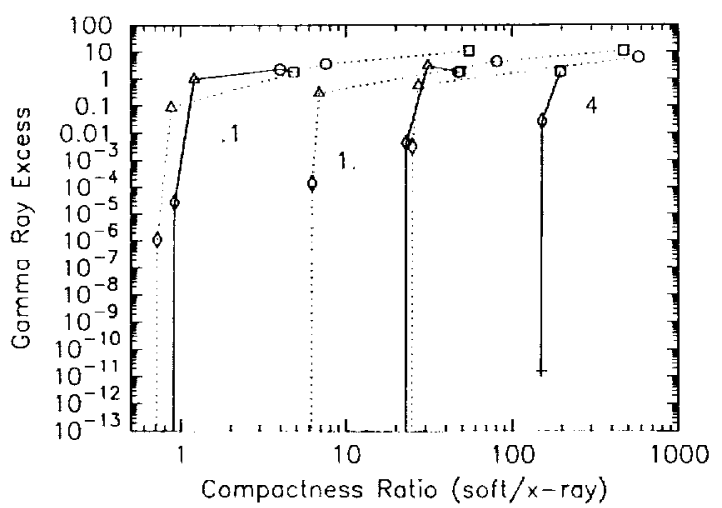

Fic. 3.-The $\gamma$-ray excess is plotted against the ratio of the sof1 to X-ray compactness parameters, $l_{s} l_{x}$, for the models in Fig. 2 . The numbers attached to pairs of solid and dotted curves represent the $l_{s} / l_{h}$ values. The squares, circles, triangles, diamonds, and crosses attached along the curves correspond to the points where $l_{f}=0.1,1,10,100$, and more than 1000 , respectively.

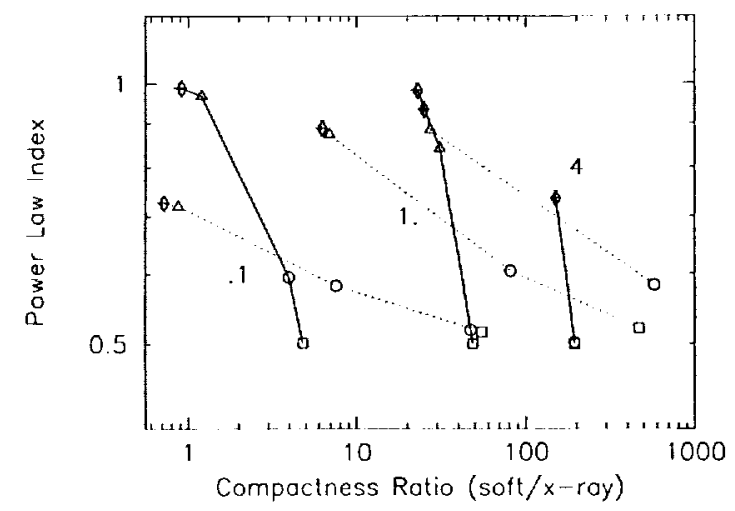

Fic. 4.- The energy index of the $2-10 \mathrm{keV}$ power-law X-ray spectrum, $\alpha_{x}$, is plotted against $l_{s} / l_{x}$ for the models in Fig. 2 . Notation is the same as in Fig.

continuum excess problem, means the production of a morc prominent $\gamma$-ray line and, consequently, makes it harder to reconcile with the lack of detection of such a line. Z90 comment that this even stronger annihilation feature predicted from their model is not observed because it will probably be broadened by motions in the emitting plasma. DF89 did estimate such broadening and applied it in their testing procedure. Z90 did not conduct any such $\gamma$-ray line testing. Obviously, however, the annihilation-line problem only becomes worse when the reflection is included. If the DF89 model fails to satisfy this line constraint, it would be cven more severe with the reflection model of Z90, where the LZ87 pair cascade is adopted. Note that the pair-cascade models of LZ87 and DF89 are qualitatively similar.

This problem, on the other hand, is solved far more naturally by introducing our disk-corona geometry. In $\$ 3$ we noted that not only the enhanced depletion of $\gamma$-ray continuum but also much weaker $0.5 \mathrm{McV}$ linc production for higher $l_{h}$ are major characteristics of our disk-corona model. The reason is that, with our choice of geometry, the primary seed photons (both $\gamma$-ray and soft) must travel through the entire column of absorbing coronal gas lying above their point of injection and extending out to around the freeze-out height $h_{f}$ beyond which there is no further change in the outcoming spectra (where $h_{f} \lesssim R_{l}$ ). (For instance, $h_{f}$ is $\sim 0.5 R_{l}$, when $l_{h}=500$.) In contrast, in HS Model the primary seed soft photons, as well as radiation injected within absorption optical depth unity of the surface of the sphere, are more or less free to escape. If $\tau_{\text {Comp }}(x)$ is the optical depth to pair creation for $\gamma$-ray energy $x$, then these $\gamma$-rays will emerge from the top of the disk-corona geometry at a rate approximately equal to the fraction $\exp \left[-\tau_{\text {Cump }}(x)\right]$ of the rate at which they injected, while for HS Model that fraction would be $\sim 1 /\left[1+\tau_{\text {Cump }}(x)\right]$ (sec KTT95). The disappearance of the $\mathcal{e}^{-}-e^{+}$annihilation line in our model at higher compactness is also due to the geometry of the model. The "life-cycle" of an $e^{-}-e^{+}$pair is creation, Compton cooling, thermalization, and annihilation. For high compactness, the cascade-creation process quickly depletes the high-energy ( $\gtrsim 1 \mathrm{MeV}$ ) photons, which (as there is no local injection) are not replenished. Soon the only source of high-energy photons is the pair-annihilation process, and the majority of pairs are in a (cooler) thermal distribution. Thus, we begin to lose our $\sim \mathrm{MeV}$ photons to down-scattcring. This reduces the nonthermal pair production. The thermal population continues annihilating, but fewer nonthermal pairs exist to replenish the distribution. The density drops, reducing the number of annihilation events. This further reduces the 
density of photons capable of creating new pairs, which accelerates the process. The population of $e^{-}-e^{+}$pairs decays exponentially, taking the annihilation-line strength along with it. For the same reason our $\tau_{\text {Cimm }}$ is less than in HS Model. For instance, our $\tau_{\text {Comp }} \sim 4$ when $l_{h}=500$ and $x_{m}=2.25 \times 10^{3}$, although it bccomes less for lower values of $x_{m}$ and $l_{h}$. Although our studies show that if the Fe line from the disk is to survive the passage through the corona it must be sufficiently strong (KTT95), that should not pose a serious problem. For instance, in reality, the disk may be "patchy," in the sense that the injection from the disk surface takes place in discrete active regions. In fact, very recently such a "patchy" disk-corona configuration had to be introduced to thermal models also, in order to make them consistent with observation (c.g., Stern et al. 1995, hereafter S95). Also, the cold component within $\sim 10 R_{g}$ may consist of not only a disk but also an assembly of blobs (or filaments) spreading over more extended regions which contribute significantly to the Fe line.

Wc are aware of the existence of various thermal diskcorona models (see, e.g., S95 and references thercin). The detailed comparison of our nonthermal disk-corona model with these thermal models, as well as detailed spectral fitting to the observational data, are outside the scope of this current short Letter. (See KTT95 and Kellen \& Tsuruta 1995a for such studies.) However, the important difference is that, in our model, the pairs which play a major role are produced nonthermally and, especially, that in most of the existing thermal models the hard photon injection and energy dissipation take place over the whole coronal region, while in our model they are confined to the base of the coronal region.

It is generally thought that radio-quiet quasars and Seyfert nuclei are powered by accretion of gas onto a supermassive black hole (sce, e.g., review by Rees 1984). The presence of relatively cool, dense plasmas is then inevitable near the central engine of these objects (Guilbert \& Rees 1988, hereafter GR88; Lightman \& White 1988, hereafter LW88). Therefore, the presence of various additional fine structures, such as a soft X-ray excess, iron and other emission-line and absorption features, and a hard X-ray reflection hump, have been predicted as a natural consequence of the reprocessing of $X$-rays by the cool component in the vicinity (GR88 and LW88). Such fine structures have been discovered by EXOSAT, Ginga, and ROSAT (e.g., Pounds et al. 1990; Matsuoka et al. 1990; Nandra \& Pounds 1994). In order to construct a composite physical model consistent with both theory and observation, this reprocessing by further reflection has to be included. The work presented here was carried out as our first step toward the construction of such a more realistic model. Further reffection of the coronal component by the cold material is currently under investigation (Kellen \& Tsuruta 1995b). However, the purpose of our current Letter is to report on our testing mainly of the $\gamma$-ray constraints. Note that in our testing procedure we self-consistently took into account the effect of reflection, and, hence, further calculations of reprocessing will not affect our major conclusion, namely, that the introduction of our disk-corona geometry to the nonthermalpair model offers a natural solution to the obsenved $\gamma$-ray constraints. The most recent COMPTEL data appear to indicate that the upper limit to the bump in the diffuse background $\gamma$-radiation is significantly reduced (e.g., Collmar 1995, private communication). However, note that most of our curves in Figures 2 and 3 (with $l_{h}>\sim 10$ ) lie comfortably below the $\gamma$-ray excess $=1$ line, so that the above conclusions will not be affected by this newer constraint (see KTT95). The latest RGO/OSSE cutoff energy constraint is $\sim 0.2<E_{\mathrm{c}}<\sim 1 \mathrm{MeV}$ (e.g., Zdziarski et al. 1995, hereafter Z95a; Madejski et al. 1995, hereafter M95; Zdziarski 1995, hereafter Z95b). In fact, currently the $200-600 \mathrm{keV}$ OSSE sensitivity is not sufficient to be able to offer any stronger constraint. In Figure 1 a typical value is $E_{c} \sim 500 \mathrm{keV}$, which is certainly within this limit. Note that this value, however, corresponds to $x_{m}=2.25 \times 10^{3}$. For larger $x_{m}$ the corresponding $E_{c}$ becomes lower. The cutoff energy may be much less for Seyfert 2 galaxies (c.g., Z95b). However, note that there has been increasing observational evidence that the spectra of Seyfert 2's are intrinsically flatter than those of Seyfert l's (e.g., Z95b and references therein), and, hence, there probably is an intrinsic difference between the dominant emission mechanisms of these two types of Seyferts. For instance, Seyfert 2 radiation is consistent with nonrelativistic thermal models (e.g., Z95b and Sunyaev \& Titachuk 1985). In fact, it could be that both thermal and nonthermal components are present, but the difference could be that in some cases (e.g., Seyfert 2's) the nonrelativistic thermal component is dominant, while in other cases (e.g., Seyfert l's) the nonthermal process is more effective.

We have demonstrated that a relevant model consistent with the $\gamma$-ray constraints on Seyfert 1 galaxies can naturally be nonthermal, when a certain realistic geometrical effect is taken into account. We emphasize that, as far as we are aware, this is the first paper in which our kind of disk-corona configuration is introduced to the nonthermal pair-cascade model and, moreover, where detailed observational testing of such a model has been carried out.

S. T. thanks many colleagues, including M. J. Rees, N. Brandt, Z. Kuncic, K. Nandra, W. Collmar, and A. Fabian, for stimulating discussions. We thank the anonymous referee for valuable comments. This work was supported partially by NASA grant NAGW-2208.
Coppi, P. S., \& Blandford, R. D. 1990, MNRAS, 245, 453

Done, C., \& Fabian, A. C. I989, MNRAS, 240,81

Fabian, A. C., Blandford, R. D., Guilbert, P. W., Phinney, E. S., \& Cuelar, L. 1986, MNRAS, 221, 931

Galeev, A. A., Rosner, R. \& Vaiiuna, G. S. 1979, ApJ, 229, 318

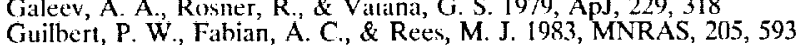

Guilbert, P. W., \& Rees, M. J. 1988, MNRAS, 233, 475

Kellen, M., \& Tsurutia, S. 1995a, in preparation

Ke.len, 1995 b, in preparation

Kellen, M., Tsuruta, S., \& Tritz, B. G. 1995, in preparation

Lightman, A. P. 1982 , ApJ, 253, 842

I.ightman, A. P., \& White, T. R. 1988, ApJ, 335, 57

Lighman, A. P., \& Zdziarski, A. A. 1987, ApJ, 319,643

Madejski, G. M., et al. 1995, ApJ, 438, 672

Matsuoka, M., Piro, L., Yamauchi, M., \& Murakami, T. 1990, ApJ, 361, 440

\section{REFERENCES}

Nandra, K., \& Pounds, K. A. 1994, MNRAS, 268, 405

Pounds, K. A., Nandri, K., Stewart, G. C., \& Fabian, A. C. 1990, Nature, 344, 132

Rccs, M. J. 1984, ARA\&A, 22, 471

Stern, B. E., Poutanen, J., Svensson, R., Sikora, M., \& Begelman, M. C. 1995, ApJ, 449, LI3

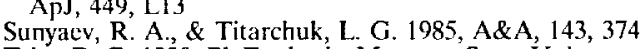

Tritz, B. G. 1990, Ph.D. thesis, Montana State Univ.

Zdziarski, A. A., 1995, Adv. Space Res., in press

Zdziarski, A. A., \& Coppi, P. S. 1991, ApJ, 376,480

Zdziarski, A. A., Ghisellini, G., Goorge, I. M., Svensson, R., Fibian, A. C., \& Done, C. 1990, ApJ, 363, Li

Zdziarski, A. A., Johnson, W. N., Done, C., Smith, D., \& McNaron-Brown, K. 1995, ApJ, 438, L63 